\section{IRON AND ALLOTROPY.}

$\mathrm{M}^{\mathrm{A}}$ NY points of theoretical interest as well as of practical importance were brought forward in the discussion "On the Transformation of Pure Iron" opened by Dr. A. E. Oxley, of the University of Sheffield, at the Faraday Society on October I9. The subjoined summary describes the main views presented.

It is now believed by many metallurgists that the $\mathrm{A}_{2}$ transformation of iron can be explained without assuming allotropic change, but the $A_{3}$ transformation is regarded as involving allotropy. The crystalline state is one of extreme molecular association (physical polymerism or the grouping of chemical molecules under the influence of mutual physical forces), and if allotropy is determined solely by the nature and extent of this association each substance will show an unlimited number of allotropic forms within a finite temperature interval. On this view the attempts to show that $A_{3}$ involves allotropic change while $A_{2}$ does not, need not have been made.

To surmount the difficulty presented by the existence of so many allotropes, two different types of allotropy have been recognised: (I) the two-phase (discontinuous) type, (2) the one-phase (continuous) type. Having adopted these, we have now to determine experimentally to which type a given transformation belongs. The burden is thus thrown on our experimental refinements, and the difficulty of drawing a sharp distinction is at once apparent-the distinction can only be arbitrary. In this connection the liquid crystalline state is interesting. There are some substances which show no liquid crystalline state on fusion, but do so prior to recrystallisation. Thus we have two-phase allotropy on heating and one-phase allotropy on cooling.

Defining allotropy as a difference of atomic structure of the chemical molecule (consistent with the transformation from oxygen to ozone, or from one isomer to another), a distinction can be drawn between allotropy and crystalline grouping. Allotropic modifications will form characteristic space lattices determined by their molecular constitutions. It does not necessarily follow that a difference of crystalline symmetry implies allotropy, for identical molecules can be packed together in different ways (Barlow and Pope. Trans. Chem. Soc., vol. 1xxxix., p. I741, I906). We must distinguish between the forces holding the molecules in a definite space lattice and those holding the atoms in the molecule.

Now on the theory of the molecular field Weiss has shown (Comptes rendus, vol. cxliv., p. 25, I907) that the so-called $\beta$ and $\gamma$ forms of iron have the same Curie constant, and $A_{3}$ is not an allotropic change point. In a later research (Journ. de Phys., vol. 1., p. 965, I9r1, and Arch. des Sciences, I913), using more recent data, and assuming that each atom possesses an integral number of magnetons. Weiss shows that the transition $\beta$ to $\gamma$ may be represented by a change from a tri-atomic to a di-atomic molecule. If, however, the magnetic particle of the $\gamma$ state consists of three molecules, and that of the $\beta$ state of two molecules, the magneton theory will still hold, but now the number of atoms in a molecule of each state is the same. This latter view seems more probable, for $\gamma$ iron possesses more magnetons per molecule than $\beta$ iron does, and therefore unless we suppose that the molecules of the $\beta$ state are so bound together that one cannot rotate without dragging along its neighbours, it seems difficult to account for the rapid increase of susceptibility on cooling through $\mathrm{A}_{3}$.

If cooling through $\mathrm{A}_{3}$ is accompanied by a closer grouping of the molecules in the direction (characteristic for each crystal) of spontaneous magnetisation, there will be no change of crystalline symmetry, while the increased interaction of the molecules will give rise to ferro-magnetism. The change of molecular distances in the perpendicular directions is not important from a magnetic point of view, each molecule being mainly constrained by the one in front and the one behind in the direction of the magnetic axis. (Silver iodide (hexagonal) is an example of a crystal which expands along the axis while contracting in perpendicular directions.) Thus this interpretation is not necessarily inconsistent with known magnetostriction data or with determinations of thermal expansion (linear or volume) of a mass of iron crystals.

The thermal evolution at $A_{3}$ is due to the readjustment of molecular distances. The rise of temperature observed is $\mathrm{I}^{\circ}$ C. (Arnold, B.A. Report, Sheffield, IgIo), and taking $O \cdot I$ as the specific heat of iron, the thermal evolution is $\mathrm{I} \cdot 4$ cals./gram. This value is small compared with the latent heat of fusion of elements, e.g. P (5), Bi (13), Cd (14), Pb (5), Ag (22), $\mathrm{Sn}$ (14), $\mathrm{Zn}$ (28), Ga (I9). Further these latent heats are in general small compared with the thermal evolutions in known allotropic and isomeric transformations. Thus

$\{96$ gr. nzone $=96$ gr. oxygen $+59,200$ gr. cals.

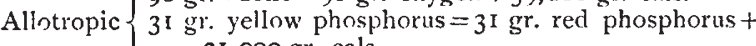
$21,000 \mathrm{gr}$. cals.

$\{78$ gr. dipropargy $l=78$ gr. benzene $+100,000$ gr. cals. Isomeric $\left\{5^{8} \mathrm{gr}\right.$. ally\} alcohol $=58 \mathrm{gr}$, acełone $+18,600 \mathrm{gr}$. cals. $=5^{8} \mathrm{gr}$. propaldehyde $+22,600 \mathrm{gr}$. cals.

The transition ${ }_{2} \mathrm{O}_{3} \longrightarrow 3 \mathrm{O}_{2}$ has the thermal value +600 cals. $/ \mathrm{grm}$. The transition $\beta \longrightarrow \gamma$ in iron has the thermal value $-\mathrm{I} \cdot 4$ cals./grm. Can we regard this latter transition as $2 \mathrm{Fe}_{3} \rightarrow 3 \mathrm{Fe}_{2}$ ?

The small thermal evolution at $\mathrm{A}_{3}$ favours the view expressed above that the transformation involves a molecular regrouping, similar to that occurring in the ordinary process of crystallisation, rather than a rebuilding of the atomic structure of the molecule. Hitherto we have not considered a change within the atom itself, such as a variation of the electric or magnetic elements. Some such change must occur in the iron atom, as it enters into different chemical combinations, otherwise how can we explain why iron carbonyl, $\mathrm{Fe}(\mathrm{CO})_{5}$, is diamagnetic, while ferrous chloride, $\mathrm{Fe}(\mathrm{Cl})_{2}$, is strongly paramagnetic? Do we not here have a kind of atomic allotropy? In this sense the allotropic theory may be consistent with the carbide theory which attributes the properties of carbon-steels to definite compounds of iron with carbon (such as cementite $\left(\mathrm{Fe}_{3} \mathrm{C}\right)$ ). Magnetic phenomena appear to be so definitelv related to the atom that the existence of different types of iron atom is suggested inevitably, and the suggestion would not be at variance with modern views of atomic structure. Many arrangements of electronic orbits in dense atoms are theoretically possible.

The work of Prof. W. H. and Mr. W. L. Brags. must be considered in relation to any phenomenon of crystals. They have shown the difficulty, even in ordinary cases, of even defining the molecules of the crystals, although in many cases this is possible. But to determine by their method whether, in an iron crystal, any atom has a special relation to one of those surrounding it, would be nearly impossible. The optical effects which they investigate are determined only by the nucleus or core of the atom, and the outer arrangements in the atom, determining its allotropic forms, might differ considerably without being capable of detection except perhaps by their magnetic properties. On these lines, therefore. no immediate objection to the existence of such different types of atom

I Vide Muir and Wilson, "Elements of Thermal Chemistry," pp. 250-53, for many uther examples.

NO. $240 T$, VOL. 96] 
san apparently be raised, and the suggestion of atomic allotropy appears worthy of development as a possible interpretation of others among the phenomena of pure iron, which considerations of space have excluded from the present summary.

During the discussion several interesting slides showing variations of the micro-structure of iron were exhibited by Prof. H. C. H. Carpenter.

\section{PREVENTIVE MEDICINE IN PENNSYLVANIA.I}

PREVENTIVE medicine is a science so likely to appeal to the genius of the American people that it is of considerable interest to read the reports issued in July, August, and September of 19I4, by the Pennsylvania State Department of Health on various aspects of its activities.

As elsewhere, so in the State of Pennsylvania, the force which caused the development of preventive medicine was the compulsion exerted by outbreaks of disease. An epidemic of typhoid fever in 1885 was the means of obtaining the enactment of a previously twice defeated Bill to establish a State Board of Health, which, however, at first was much circum. scribed in its powers and duties. A severe outbreak of smallpox in the years 190I-04 impelled the Legislature to establish a State Department of Public Health.

At its head is a Conmissioner of Health, with very great powers of initiative, on whom falls the duty of appointing whatever assistants he may find necessary to carry on the work. The State Department of Health has direct executive control over all public health problems of every sort in the more rural portions of the State, comprising four-fifths of the land area and one-quarter of the total population. Over the remainder of the population, aggregated in the more densely populated townships, which are required to maintain their own local boards of health, the State Department of Health has advisory and supervisory control, but no executive responsibility except in relation to sanitary engineering, tuberculosis, and the collection of vital statistics.

The State administrative machinery, therefore, in some respects resembles, but in others is in marked contrast to, that of an English county health department. Both have general supervisory and advisory duties with regard to the smaller areas within their compass. The English county department has, however, nothing approaching to the wide general executive functions of the Pennsylvania State Department, although in some respects-for example, in the control of tuberculosis-their activities are akin. The complete responsibility of the Pennsylvania State Department for the health of the public in the rural portions of its area has no analogy in county administration in this country. The English system is based far more than is the Pennsylvanian on local as opposed to central administration.

The organisation of the State Health Department would appear to be on very effective lines. Out of the central department have crystallised a number of subdepartments, or divisions, dealing respectively with vital statistics, school medical inspection, sanitary engineering, tuberculosis dispensaries and nursing, sanatoria, housing, laboratories, and the distribution of biological products. The chief of each division reports directly to the health commissioner. 'There are two special divisions, one of which conducts all the auditing and accounting and part of the purchasing for other divisions, while the other attends to the whole of the storage and shipment of materials. The

1 Pennoylvania Health Bulletin, July, August, September, 19r4. NO. 240I, VOL. 96$]$ divisional executive officers are thus left free to devote their energies to the more essential public health work. Comparison of the work of some of these divisions with that of health departments in this country is instructive. For example, the registration of births and deaths is far more closely bound up with public health in Pennsylvania than is the case in England and Wales, in which it is not in any way directly controlled by the Health Department; whereas in Pennsylvania, on the contrary, the Division for Vital Statistics, a branch of the State Health Department, under the direct supervision of a health officer, is responsible for precisely the same work as is done by our local and central registrars in respect of births and deaths and marriages.

In the same way the school medical service, instead of being, as is unfortunately the case in this country, a separate service affiliated only for reasons of convenience to the public health service, is recognised in Pennsylvania as essentially a branch of public health, and as such is administered by the State Health Department through a division of medical inspection. Sanitary engineering, again, carried out as it is by a special division of the State Health Department, is much more clearly recognised as a function of public health than is the case in this country.

Perhaps the most striking feature of the Pennsylvanian scheme is its systematic educational campaign. All reports sent in from every division of the Health Department to the Health Commissioner are dealt with from their public health aspect, and are then handed over to an educational section of the central department, where they are rewritten and issued in popular form as lectures, circulars, leaflets, newspaper talks, or periodical bulletins. This eagerness to popularise technical knowledge is an important side of public health work, which could with advantage be better developed in this country. In general administration, probably English methods give as good results, though possibly in more circuitous ways. Pennsylvania has had the advantage of beginning at a time when it could develop its public health system at one stroke and as a whole, and so could largely avoid the errors and vagaries of a system which has grown by accretion.

H. P. N.

\section{CHEMISTRY AT THE BRITISH ASSOCIATION.}

A LTHOUGH so many topical subjects were, on $A$ account of the war, excluded from the discussions of the section, the attendance at the meetings was on the whole satisfactory, exceeding the anticipations of the earlier part of the year. It is, however, clear that anything like general interest can only be cvoled in the Chemical Section by discussions on rather broad subjects, papers giving the results of researches on some particular branch being only too frequently delivered to nearly empty benches. This not infrequently arises from the paper being so specialised that the author is practically the only person in the room capable of appreciating its significance and value. One cannot help feeling that the atmosphere of the Chemical Society or other specialised body would be more sympathetic. Under the exceptional circumstances of this year foreign guests were few in number, but the section had the pleasure of welcoming and listening to two of our Belgian allies-Prof. Henry and Prof. Ranwez, from Louvain, the former giving an account of researches on the preparation and properties of vinylacetic nitrile, which he has carried out in Prof. Perkin's laboratory at Oxford during his residence in this country, while the latter contributed a paper to the discussion on smoke. 